\title{
Special Review
}

\section{Role of the Ionosphere for the Atmospheric Evolution of Planets}

\author{
MASATOSHI YAMAUCHI and JAN-ERIK WAHLUND
}

\begin{abstract}
We have synthesized current understanding, mainly observations, with regard to ion escape mechanisms to space from the ionosphere and exosphere of Titan and Earth-type planets, with the intent to provide an improved input for models of atmospheric evolution on early Earth and Earth-type planets and exoplanets. We focus on the role of the ionosphere and its nonlinear response to solar parameters, all of which have been underestimated in current models of ancient atmospheric escape ( 4 billion years ago). Factors that have been overlooked include the following: (1) Much larger variation of $\mathrm{O}^{+}$outflow than $\mathrm{H}^{+}$outflow from the terrestrial ionosphere, depending on solar and geomagnetic activities (an important consideration when attempting to determine the oxidized state of the atmosphere of early Earth); (2) magnetization of the ionopause, which keeps ionospheric ions from escaping and controls many other escape processes; (3) extra ionization by, for example, the critical ionization velocity mechanism, which expands the ionosphere to greater altitudes than current models predict; and (4) the large escape of cold ions from the dense, expanded ionosphere of Titan. Here we offer, as a guideline for quantitative simulations, a qualitative diagnosis of increases or decreases of non-thermal escape related to the ionosphere for magnetized and unmagnetized planets in response to changes in solar parameters (i.e., solar EUV/FUV flux, solar wind dynamic pressure, and interplanetary magnetic field). Keywords: $\mathrm{O} / \mathrm{H}$ ratio-Atmospheric escape-Planetary evolution-Ionosphere-EUV/FUV-Solar wind. Astrobiology 7, 783-800.
\end{abstract}

\section{INTRODUCTION}

C ONSTRUCTING RELIABLE MODELS of the ancient atmosphere and its early evolution is one of the key issues in studies of the origin and evolution of early life on Earth. It is also key to understanding habitable conditions with regard to exoplanets. Past efforts have focused on modeling an initial neutral atmosphere from planetary formation models (e.g., Owen, 1998; Kasting and Brown, 1998; Abe et al., 2000; Commeyras et al., 2006; Hashimoto et al., 2007). Only recently have efforts to model the atmospheric evolution (e.g., escape, migration, and supply) of ancient Earthtype planets received serious attention in this astrobiological framework (e.g., Kasting and Catling, 2003; Lammer et al., 2003, 2007; Lundin and Barabash, 2004; Ribas et al., 2005; Kulikov et

Swedish Institute of Space Physics, Kiruna, Sweden. 
al., 2006; and references therein). Main destinations of atmospheric loss are to space (escape) and to the ground (migration through rain washing, freezing, and sublimation, and chemical reactions to form $\mathrm{CaCO}_{3}, \mathrm{CH}$-group, graphite, etc.) (Kasting and Brown, 1998; Kasting and Catling, 2003; Lammer et al., 2003; Hashimoto et al., 2007).

Unfortunately, our knowledge of both initial conditions and evolution is quite limited, which has resulted in a large variety of models. Even the density of the initial terrestrial neutral atmosphere can differ by as much as two orders of magnitude between models, and this ambiguity becomes even more severe after different evolution models are applied. The large ambiguity with regard to atmospheric evolution is largely due to our lack of understanding of atmospheric escape into space. For example, thermal (Jeans) escape, one of the most important types of escape from early Earth, is derived from a simple formula using temperature, but its effect in terms of the oxidation history of the atmosphere is still difficult to estimate. This is because (a) it is not easy to estimate the exobase temperature (which determines the Jeans escape rate) for assumed extreme-ultraviolet (EUV) and far-ultraviolet (FUV) flux, unless we know exactly the cooling effect by, for example, $\mathrm{CO}_{2}$ (Tian et al., 2005, 2006; Kulikov et al., 2007); and (b) even a small ambiguity in the exobase condition causes large ambiguity in the $\mathrm{O} / \mathrm{H}$ ratio of escape because Jeans escape is selective to light elements $(\mathrm{H}$ and $\mathrm{He}$ for Earth). The change of the oxidation state of the atmosphere from the time of atmospheric/ocean formation to the time of life emergence and its early evolution is among the most-needed information.

Non-thermal ion escape, which has a different $\mathrm{O} / \mathrm{H}$ ratio from thermal escape and is much more difficult to model than thermal escape, adds another large ambiguity to the atmospheric model. As described in the following sections, observations of different planets at different solar cycle phases show a larger variability of non-thermal ion escape than is adopted in existing models within the current variation level of solar radiation and solar wind parameters. Furthermore, a large escape flux of heavy cold ions has recently been discovered around Titan, despite the low solar luminosity there (Wahlund et al., 2005). These unexpected observations indicate that many processes of atmospheric escape to space over solar system history are still poorly understood and may be underestimated.
Thus, thermal and non-thermal escape processes make the already ambiguous characterizations of atmospheric properties (such as the oxidization state) even more ambiguous when life emerged and was stabilized. To reduce such ambiguities in models, it is important to address the atmospheric loss processes on early Earth 4.5 to 3.8 billion years (Gyr) ago by broadly synthesizing current observations of elementary processes on Earth-type planets (e.g., Earth, Mars, Venus, and the moon Titan).

Although the escape mechanisms that occur on magnetized planets (e.g., present Earth) and on unmagnetized planets (e.g., present Venus, Mars, and the moon Titan) are different from each other (cf. Fig. 1), syntheses of escape mechanisms for both magnetized and unmagnetized planets are essential to our understanding of early Earth because we do not know whether early Earth was magnetized or unmagnetized at the time of the emergence of life. There is no evidence of magnetism in the rock record from 4 Gyr ago, at which time the Earth's dynamo may not have been very strong if the solid-state inner core was not fully formed. Recent paleomagnetic results indicate that the geomagnetic field 3 Gyr ago was much weaker than the present field (Sumita et al., 2001). Thus, the strength of the internal magnetic field 4 Gyr ago is an unknown parameter. Furthermore, the ionospheric height at early Earth is expected to have been higher than at present due to the high EUV/FUV flux (Evans, 1977; see also textbook by Rees, 1989), and it could have been even higher than the magnetopause height if the geomagnetic field was relatively weak. In this case, ancient Earth could be regarded as an unmagnetized planet in terms of the interaction with solar wind and the interplanetary magnetic field (IMF).

A synthesis of planetary and Earth observations helps to highlight overlooked or underestimated processes in existing models. Unfortunately, few papers are devoted to such an effort; hence it is urgent to address this problem so that ongoing modeling efforts do not neglect to take into account important processes. Here we focus on one such overlooked element, the ionosphere and its varying roles as (a) a source of escaping ions with a higher $\mathrm{O} / \mathrm{H}$ ratio than thermal escape, (b) a shield by which to prevent the loss of ions from solar wind-driven escape, and (c) a controller of large-scale interactions between solar 


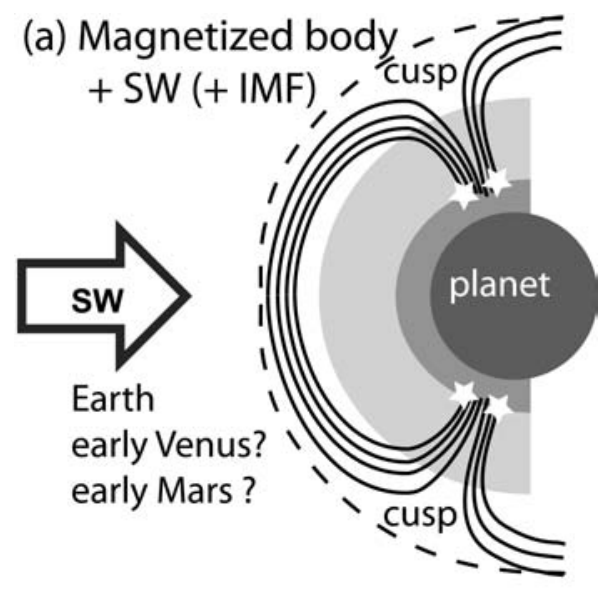

(b) Unmagnetized body

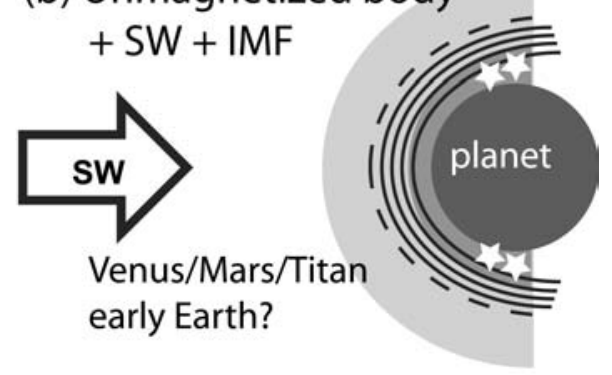

(c) Unmagnetized body

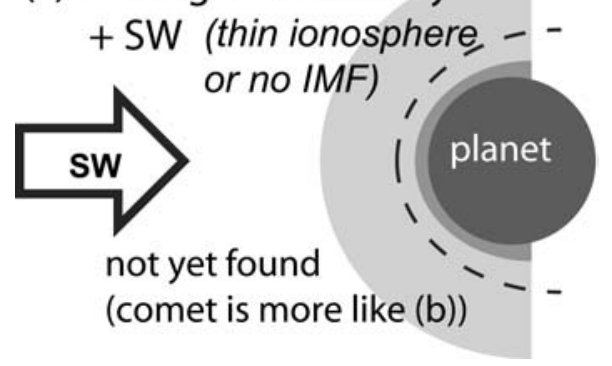

FIG. 1. Illustration of solar wind (SW) interaction with a magnetized planet and with an unmagnetized planet with a substantial atmosphere (see section 3 for details) downstream of the bow shock. Black, dark gray, and light gray areas denote the solid planet, ionosphere, and exosphere, respectively. The solid lines denote the magnetic field, and the dashed line denotes the shielding boundary (magnetopause for a magnetized planet and ionopause for an unmagnetized planet) that stops the shocked solar wind. The white stars denote the region where non-thermal (electromagnetic) ion heating is observed in the dayside ionosphere at both Earth and Mars. (a) A magnetized planet. (b) An unmagnetized planet. For Titan, one must replace SW and IMF with the saturnian magnetospheric convection and magnetic field for most of the time. (c) For reference, we add a situation when the ionospheric pressure is much lower than the SWDP. In this case, the shocked solar wind is stopped by the aerodynamic pressure of the atmosphere. The same situation is expected when there is no magnetic field anywhere, though this is unrealistic. wind and the planetary magnetosphere/ionosphere. The significance of these varying ionospheric roles for both magnetized and unmagnetized planets depends on conditions on the Sun and attributes of the resultant solar wind.

The main goal of this paper is to examine the influence of the ionosphere on atmospheric escape using current observational knowledge. We have synthesized relevant observations in order to show how the ionospheric roles are underestimated (section 2), and we have formed an overview of the geometry (section 3). Further, we have considered qualitative prognoses of how the atmospheric escape processes related to the ionosphere respond to drivers such as solar wind parameters (section 4) and the solar EUV/FUV flux (section 5). As described below, the overall dependence of atmospheric escape on some solar parameters can even be the opposite of what is currently believed, if the contribution from the ionosphere is properly estimated.

\section{THE ESCAPE TO SPACE}

The major escape mechanisms of atmospheres (from both magnetized and unmagnetized planets) known to date are summarized in Table 1. The kinetic energies of non-thermal ion escape mechanisms [(4)-(6)] are ultimately supplied by the solar wind, though solar EUV /FUV radiation largely influences the ionization. For further explanation of these mechanisms, see Lundin et al. (1991), Brace and Kliore (1991), Luhmann and Bauer (1992), Luhmann et al. (1992), Lundin and Barabash (2004), Lammer et al. (2003, 2005a, 2005b), and Kulikov et al. (2006) for unmagnetized planets, and Kasting and Brown (1998), Moore et al. (1999), Sibeck et al. (1999), Kasting and Catling (2003), and Fujimoto et al. (2006) for magnetized planets.

\subsection{Controlling variables}

One major problem in modeling the atmospheric escape on ancient Earth is that there are many controlling parameters whose values are unknown, such as (a) solar EUV/FUV intensity; (b) solar wind dynamic pressure (SWDP) or, more precisely, the solar wind density, velocity, and temperature; (c) IMF for both average magnitude and its variability in direction; (d) lower 
Table 1. Major Escape Mechanisms of Planetary Atmospheres to Space

\begin{tabular}{|c|c|c|c|}
\hline No. & Type & Mechanism & Explanation \\
\hline (1) & $\begin{array}{r}\text { Thermal, } \\
\text { neutral }\end{array}$ & Jeans escape & $\begin{array}{l}\text { Thermal tail exceeds the escape velocity. } \\
\text { Principal works for ions, but they are mostly } \\
\text { trapped by magnetic field. }\end{array}$ \\
\hline (2) & $\begin{array}{l}\text { Thermal, } \\
\text { neutral/ion }\end{array}$ & Hydrodynamic blow off & $\begin{array}{l}\text { Same as the solar wind formation } \\
\text { mechanism (works under extreme radiation } \\
\text { conditions during early Sun or star } \\
\text { conditions). }\end{array}$ \\
\hline (3) & $\begin{array}{l}\text { Thermo- } \\
\text { chemical, } \\
\text { neutral }\end{array}$ & Photochemical heating & $\begin{array}{l}\text { Release of energy in the excited state gives } \\
\text { the atom escape velocity. }\end{array}$ \\
\hline (4) & $\begin{array}{l}\text { Thermal and } \\
\text { non-thermal } \\
\text { combined, } \\
\text { ion }\end{array}$ & $\begin{array}{l}\text { Ion pickup and subsequent } \\
\text { secondary sputtering of } \\
\text { neutrals by picked-up ions }\end{array}$ & $\begin{array}{l}\text { Ions that are newly exposed to solar wind } \\
\text { start moving according to the } \\
\text { electromagnetic force of the solar wind. }\end{array}$ \\
\hline (5) & $\begin{array}{l}\text { Non-thermal, } \\
\text { ion }\end{array}$ & $\begin{array}{l}\text { Non-thermal ion heating } \\
\text { by electromagnetic waves } \\
\text { and field-aligned } \\
\text { potentials }\end{array}$ & $\begin{array}{l}\text { Solar wind energy impeded locally to low } \\
\text { altitudes causes electromagnetic } \\
\text { disturbances that energize ionospheric ions } \\
\text { by, e.g., the ion cyclotron resonance } \\
\text { mechanism. }\end{array}$ \\
\hline (6) & $\begin{array}{l}\text { Non-thermal, } \\
\text { ion }\end{array}$ & $\begin{array}{l}\text { Large-scale momentum } \\
\text { transfer including all } \\
\text { processes such as } \\
\text { instability-related transfer } \\
\text { and reconnection }\end{array}$ & $\begin{array}{l}\text { The solar wind dynamic pressure and } \\
\text { electromagnetic forces push the planetary } \\
\text { plasma anti-sunward at the boundary region } \\
\text { where the solar wind is stopped by the } \\
\text { planetary ionosphere/atmosphere. }\end{array}$ \\
\hline
\end{tabular}

aSeveral mechanisms exist for the initial energization and the subsequent outward acceleration.

atmosphere composition and density; (e) the amount of heating at the surface by, for example, solar radiation and volcanism; (f) strength of the internal magnetic field; and (g) external bombardment. Among those, only the solar radiation parameters (EUV/FUV and total luminosity) are rather well estimated from the study of the history of G-type stars (e.g., Ayres, 1997; Ribas et al., 2005; Kulikov et al., 2006; and references therein). While the total luminosity of the young Sun is believed to have been $30 \%$ less than the present Sun, the EUV/FUV flux was most likely very high (factors of 10-1000) 4-4.5 Gyr ago, which would have resulted in a hot and expanded exosphere and a subsequent high loss rate due to Jeans escape (e.g., Luhmann and Bauer, 1992; Lammer et al., 2003; Kulikov et al., 2006). However, the values of the other parameters are not well defined, as mentioned in the introduction.

Note that the strength of the IMF and the solar wind are unknown parameters of early Earth. Although the solar dynamo may have been strong 4-4.5 Gyr ago because the solar rotation is believed to have been faster at that time than at present (Ayres, 1997; Ribas et al., 2005; Kulikov et al., 2006), this is not sufficient evidence to assume a strong IMF, unless the actual dynamo mecha- nism is known. Since the convection zone of the Sun was located much deeper 4-4.5 Gyr ago, the solar dynamo likely differed from its current state. We cannot assume an eleven-year solar cycle for the early Sun, and the variability of the IMF (both direction and strength) is completely unknown.

For the SWDP, Wood et al. (2005) and Wood (2006) measured the size of the astrosphere (the stellar counterpart of the heliosphere) by $\mathrm{H} \alpha$ absorption for a total of eleven examples, including the Sun (the size of the astrosphere is expected to be related to the stellar wind dynamic pressure). Together with the X-ray intensity measurements, they derived a general tendency that hotter Gtype stars have larger mass loss rate. However, this relation does not hold for very young (very hot) stars. In other words, the SWDP 4 Gyr ago is an unknown parameter, as is the IMF.

Thus, the driving parameters are mostly quite unknown. These ambiguities are further enhanced when estimating the escape rate because the dependencies of the escape processes on these parameters are not direct but indirect with regard to the shape, height profile, temperature, and dynamics of atmospheric regions (exosphere, ionosphere, homosphere, and magnetosphere if ap- 
plicable). For example, the thermospheric/exospheric temperature (a key parameter for Jeans escape) is affected by many factors, such as (a) heating through solar EUX/FUV, (b) vertical transport (e.g., Vidal-Madjar, 1978; Gordiets et al., 1982; Tinsley et al., 1986; Kasting and Brown, 1998; Kasting and Catling, 2003; Kulikov et al., 2006), (c) cooling by infrared vibrational-rotational bands of molecules (Tian et al., 2005; Kulikov et al., 2007), and (d) ionization loss of selectively superthermal atoms/ molecules with velocities beyond Alfvén's critical ionization velocity (CIV) (Stenbaek-Nielsen et al., 1990; Peratt and Verschuur, 2000; and references therein). Among these, only the direct heating by the EUV/FUV is relatively well known.

The vertical transport sets the upper limit of the atmospheric escape related to neutral atoms/molecules (e.g., the pickup loss, Jeans escape, and photochemical escape) because the removed neutrals are continually supplied from the homopause by this transport. The vertical transport is also essential in modeling the exosphere and ionosphere. However, this transport is quite difficult to model because it includes both diffusion processes (Vidal-Madjar, 1978; Kasting and Brown, 1998) and the vertical wind, the latter of which has been ignored in most models.

The cooling effect is another controversial element. It is widely believed that a strong cooling of some part of the upper atmosphere (above the tropopause), or what is called the greenhouse effect, may be a solution to the so-called early Sun paradox [i.e., liquid water should have existed when solar luminosity was 30\% less than the present value (Sagan and Mullen, 1972; Pollack, 1991; Sagan and Chyba, 1997; Kasting and Catling, 2003; Commeyras et al., 2006)]. However, the amount of greenhouse gases in existing models of the initial atmosphere is not sufficient to explain this paradox on its own (e.g., Shaviv et al., 2003; Wood et al., 2005; Commeyras et al., 2006), which indicates that there is a large ambiguity regarding the greenhouse effect, including the amount of greenhouse gases at the exobase. Therefore, it is not unreasonable to assume strong cooling in the exosphere (Tian et al., 2005; Kulikov et al., 2007). Inversely, the amount of greenhouse gases could also be small in supplemental scenarios, e.g., by introducing low albedo of clouds (Shaviv et al., 2003; see also Lassen and FriisChristensen, 1995; Svensmark and Friis-Christensen, 1997) or by modification to the solar evolution model (Wood et al., 2005).
The CIV has never been discussed in the escape processes, though this mechanism has been confirmed by ionospheric rocket experience. According to the CIV concept, a neutral (atom or light molecule) traveling with more kinetic energy relative to the background magnetized plasma than ionization energy can be spontaneously ionized by losing corresponding kinetic energy (StenbaekNielsen et al., 1990; Peratt and Verschuur, 2000; and references therein). Since the ballistic escape energy from Earth and Venus (corresponding to $11 \mathrm{~km} / \mathrm{s}$, or about $10 \mathrm{eV}$ for oxygen atoms) is comparable to the ionization energy (particularly for the excited-state atoms), the CIV ionization for a hot exosphere cannot be ignored. The CIV mechanism is just one example of underestimated ionization processes described below.

The general effectiveness of the ionization processes is one of the most important factors for all non-thermal ion escape mechanisms [(4)-(6) in Table 1], though Cassini observations indicate that this fundamental problem is not well understood yet. Cassini found that Titan's ionosphere has dense, cold, heavy ions and is expanded to $\sim 1400 \mathrm{~km}$, i.e., to the same height as or even higher than the exobase as illustrated in Fig. 1b (Wahlund et al., 2005). Since Titan has a smaller gravity and receives much less solar EUV/FUV flux than Mars, the ionospheric height has been expected to be lower than the exobase as illustrated in Fig. 1c (Cravens et al., 2005; Ågren et al., 2007). Even after including the electron impact ionization by saturnian magnetospheric energetic electrons and the drag effect by magnetospheric convection, the models predict a more compressed ionosphere than Cassini observed (Cravens et al., 2005). This observation raised questions about the present understanding of formation and dynamics of the ionosphere.

\subsection{Present knowledge of escape}

Except for hydrogen and helium, non-thermal ion escape [see (4)-(6) in Table 1] observed on any Earth-sized planet is much larger than the estimated Jeans escape (e.g., Lundin and Dubinin, 1992; Cully et al., 2003; Wahlund et al., 2005; and references therein). We begin with escape related to non-thermal ion heating [(5) in Table 1], for which statistics over a solar cycle are available for Earth.

The observed ion flux that escapes directly from the terrestrial upper atmosphere/iono- 
sphere by electromagnetic/electrostatic non-thermal ion heating is on the order of $10^{25 \sim 26}$ ions/s or $10^{0 \sim 1} \mathrm{~kg} / \mathrm{s}$ (Moore et al., 1999; Cully et al., 2003), whereas the Jeans escape of hydrogen is estimated (no direct measurement exists) to be larger than this non-thermal escape of protons during solar minimum and smaller during solar maximum, with an upper limit of $10^{27}$ atoms / s limited by diffusion (Vidal-Madjar, 1978; Kasting and Catling, 2003). The largest source of non-thermal ion escape is the dayside polar region (e.g., Kondo et al., 1990; Norqvist et al., 1998), where solar wind can directly access the ionosphere through the geomagnetic field (the "cusp" in Fig. 1a).

Table 2 summarizes current knowledge of the non-thermal ion budget above the Earth's ionosphere [related only to mechanism (5) in Table 1] during the solar maximum as observed by satellites (Moore et al., 1999; Cully et al., 2003; Yamauchi et al., 2005b). The outflow fluxes of $\mathrm{H}+$ and $\mathrm{O}+$ in Table 2 correspond to about $0.03 \sim 0.15$ $\mathrm{kg} / \mathrm{s}$ and $0.3 \sim 5 \mathrm{~kg} / \mathrm{s}$, respectively; most of the escaping mass flux is carried by heavy ions such as $\mathrm{O}^{+}$, and these non-thermal escapes are strongly correlated to the magnetospheric activity. For reference, the current estimate of incoming meteor mass flux $(0.5 \mathrm{~kg} / \mathrm{s})$ is comparable to the mass flux of ion outflow during quiet periods. The $\mathrm{O}^{+}$escape is larger than the $\mathrm{H}^{+}$escape even in its number flux during geomagnetic active periods (magnetic storms and substorms). A substantial portion of these escaping ions is, however, believed to return to the ionosphere in various forms (McFadden et al., 2003; Yamauchi et al., 2005a), probably due to the geomagnetic field. Unfortunately, the amount of this return flow is unknown except for $\mathrm{H}^{+}(0.003-0.02 \mathrm{~kg} / \mathrm{s})$, which is estimated as only one tenth of non-thermal $\mathrm{H}^{+}$ escape at $3 \sim 5$ earth radii $\left(R_{E}\right)$.

Escape of the heavy ions $\left(\mathrm{O}^{+}\right.$and $\left.\mathrm{N}^{+}\right)$increases much more than $\mathrm{H}^{+}$during high solar activity (high $\mathrm{F}_{10.7}$ flux) and during major magnetic

Table 2. Budget of Non-Thermal IONS ABOve EARTH's IONOSPHERE ${ }^{\mathrm{a}}$

\begin{tabular}{lcc}
\hline Species & $H^{+}$ & $\mathrm{O}^{+}$ \\
\hline Out $\left(\mathrm{h}=2 \sim 4 \mathrm{R}_{\mathrm{E}}\right)^{\mathrm{b}}$ & $0.2 \sim 0.8 \times 10^{26} / \mathrm{s}$ & $0.1 \sim 2 \times 10^{26} / \mathrm{s}$ \\
In $(\mathrm{h}=840 \mathrm{~km})$ & $0.02 \sim 0.1 \times 10^{26} / \mathrm{s}$ & $?$ \\
\hline
\end{tabular}

\footnotetext{
aRange is between geomagnetic quiet time and active time.

${ }^{b}$ Dynamics Explorer-1 data during solar maximum.

Abbreviations: $h$, height; $R_{E}$, Earth radii.
}

storms (Chappell et al., 1982; Hamilton et al. 1988; Cully et al., 2003). Using more than eleven years' satellite ion data for the $<70 \mathrm{eV}$ range at an altitude of $6000-10000 \mathrm{~km}$, Cully et al. (2003) concluded that non-thermal $\mathrm{O}^{+}$outflow from the ionosphere increases by a factor of $10^{2}$, while nonthermal $\mathrm{H}^{+}$outflow increases only by a factor of 3 when the $F_{10.7}$ flux increases by a factor of 3 (from solar minimum to solar maximum). They also showed a stronger dependence of $\mathrm{O}^{+}$than $\mathrm{H}^{+}$on the geomagnetic activity that is controlled by the solar wind parameters, particularly, the SWDP, IMF strength, and IMF variability. This strong dependence of $\mathrm{O}^{+}$escape (for both escape energy and flux) on the solar wind parameters is also confirmed by hot plasma $(30-30,000 \mathrm{eV})$ data above the escape velocity (Arvelius, 2005).

For observations of Mars and Venus, we cannot distinguish between escape due to non-thermal ion heating and bulk momentum transfer [(5) and (6) in Table 1]. The non-thermal escape of heavy ions from the venusian atmosphere is detected as an "ionotail" or "detached plasma cloud" (observation by cold plasma instrument), with total escaping $\mathrm{O}^{+}$flux of $0.1 \sim 2 \mathrm{~kg} / \mathrm{s}$, a factor of 20 change between solar minimum and solar maximum (e.g., Brace et al., 1990; and references therein). For Mars, the relevant escape [(5) and (6) in Table 1] is reported to be about $0.1 \sim 1$ $\mathrm{kg} / \mathrm{s}$ for the solar maximum from Phobos-2 (Lundin et al., 1990; Lundin and Dubinin, 1992) and $0.02 \sim 0.1 \mathrm{~kg} / \mathrm{s}$ for the solar minimum from Mars Express (Lundin et al., 2004; Barabash et al., 2007). Since Mars Express covers only high energy (>about $300 \mathrm{eV}$ ) and Phobos-2 covers both high and low energies, this difference might be due to the instrumental difference, and we will have to wait for the Mars Express result from solar maximum to confirm the solar cycle variation.

The ion pickup process [(4) in Table 1] is a thermal and non-thermal combined process, in which the interplanetary electric field (perpendicular to the solar wind direction) and IMF move all newly born ions inside the solar wind anti-sunward (trajectories are cycloid). Even neutrals at zero velocity (well below the escape velocity) under the hydrostatic equilibrium in the gravity field escape through this process when they are exposed to solar wind and subsequently ionized by, for example, solar FUV, electron impact, and charge exchange. This process is considered to have contributed substantially to the ancient atmospheric loss from early unmagnetized planets in the ex- 
isting models (e.g., Luhmann and Bauer, 1992; Lammer et al., 2005a; Kulikov et al., 2006) for both light $(\mathrm{H}$ and $\mathrm{He})$ and heavy $\left(\mathrm{O}\right.$ and $\left.\mathrm{CO}_{2}\right)$ species. At present Mars, the observed pickup proton flux of exospheric origin is as high as $1 \sim 2 \%$ of the solar wind flux (Yamauchi et al., 2006), which corresponds to a total escaping flux from Mars of $10^{25 \sim 26} / \mathrm{s}$ or $0.02 \sim 0.1 \mathrm{~kg} / \mathrm{s}$ and is less than, but still comparable to, the calculated Jeans escape (Lammer et al., 2005a).

For a magnetized planet, the pickup loss of the exospheric neutrals is very little because the exosphere does not extend much above the magnetopause. However, the ion pickup process itself still works for ions that are kept supplied to the solar wind from the ionosphere. In fact, a large number of ionospheric ions are found in the solar wind at Mars (Lundin et al., 1991; Lundin and Barabash, 2004) and at Earth (Eklund et al., 1997; Rème et al., 2001), and they should be lost even if their velocity is less than the escape velocity. These massive ions also cause a mass loading effect, which enhances the solar windmagnetosphere interaction and subsequent largescale ion escape (Yamauchi and Lundin, 1997; Yamauchi et al., 2003).

The observation of an unexpectedly thick and dense Titan ionosphere (section 2.1) showed another form of massive ion pickup processes by Saturn's magnetospheric convection rather than by solar wind (Wahlund et al., 2005; Ågren et al., 2007). Cassini detected that a substantial amount of cold ionospheric ions is exposed to the reduced magnetospheric convection above the "soft" ionopause ( $c f$. Fig. 1b, but the convection gradually decreases toward the ionopause). Cold ions have negligibly small gyroradii; hence their trajectory is no longer cycloid but laminar along the magnetospheric convection (in the $\mathbf{E} \times \mathbf{B}$ direction). The resultant massive escape of cold ions means that they are kept supplied from the ionosphere below the ionopause.

\subsection{Importance of the ionosphere}

Thus, the observations from Earth, Mars, and Titan indicate that non-thermal escape from the ionosphere cannot be ignored in both its total amount and its role in determining the oxidizing state of the atmospheres of early Earth, Venus, and Mars. However, no existing model of atmospheric escape from early Earth (and Earth-type planets) has included the strong solar parameter dependence of non-thermal escape selectively on $\mathrm{O}^{+}$. No model has included the extra ionization by, for example, the CIV mechanism. No model has included any mechanism that explains the massive ion escape at the extended ionosphere of Titan. Furthermore, no model has properly included the solar parameter dependence of the ionopause location, which demarcates the type of loss process that is discussed later in this paper. In other words, the roles of the ionosphere and the solar wind-ionosphere interaction have been underestimated in past models of atmospheric escape from ancient Earth.

Therefore, it is important to consider the role of the ionosphere, i.e., how the basic controlling parameters (solar parameters and internal parameters) affect the various loss mechanisms through the ionosphere and its interaction with the solar wind. Unless we quantify and understand these effects, we cannot properly model the atmosphere of early Earth and Earth-type planets and exoplanets.

At present, even the ionospheric density can be underestimated in models of ancient atmospheric escape. For example, most models of ancient atmospheric escape do not include extra ionization (see Titan observation in section 2.1), which eventually locates the ionopause above the exobase (e.g., Kulikov et al., 2006). Furthermore, they calculate the ionosphere only above the altitude of peak electron density with constant UV/electron impact ionization rate (e.g., Ma et al., 2004). Only recently, Brecht and Ledvina (2006) considered the UV effect on ionization from a fixed neutral profile in their hybrid code simulation to estimate the ion escape, but they suggested a need to improve the code to obtain a more accurate boundary location.

Among the many possible parameters that affect ionospheric/exospheric conditions, we only consider in our qualitative assessment the solar parameters, which include dependence on solar EUV/FUV (which determines the ionospheric thickness), on SWDP, and on IMF (intensity and variability). Note that the magnetospheric activity (which is related to the non-thermal escape of ionospheric ions) of Earth is known to be strongly controlled by the IMF direction, which changes very frequently (e.g., textbook by Akasofu, 1977). Therefore, the variability in the IMF direction is also an important controlling factor. Luhmann and Bauer (1992) have already pointed out the importance of this type of consideration, but the 
roles of the ionosphere have not been well integrated into previous models.

As discussed in section 2.1, many indirect controlling factors are not completely understood, and our current knowledge on controlling parameters is simply insufficient for solid modeling of the ancient terrestrial atmosphere. Therefore, it is advisable to examine qualitatively one element (the ionosphere), i.e., provide an overview of the qualitative dependence of escape processes related to the ionosphere on the essential parameters. We examine only the increase or decrease of atmospheric escape through the change of ionospheric condition by increasing the unknown solar parameters.

Since solar wind dependencies are qualitatively different between the magnetized planets (e.g., Earth) and unmagnetized or weakly-magnetized planets (e.g., Venus, Mars), as shown in Fig. 1, we first briefly overview solar wind interaction with magnetized and unmagnetized planets in section 3. Then, solar wind (SWDP and IMF) dependence is considered in section 4 , and solar EUV/FUV dependence is considered in section 5.

\section{SOLAR WIND INTERACTION WITH THE IONOSPHERE}

For a magnetized planet (Fig. 1a), solar wind is deflected by the planetary magnetic field (SWDP is balanced with the magnetic pressure of the planetary magnetic field), except in singular regions where the compressed IMF (after bow shock) and the compressed planetary magnetic field (by SWDP) cancel each other (e.g., Alfvén and Fälthammar, 1963). The boundary that deflects solar wind is called the magnetopause, and the singular region is called the cusp. The cusp is magnetically connected to the ionosphere through a vertically pointing magnetic field. When the planetary magnetic field is approximately dipolar, cusps appear near the north and south poles of the dipole field.

Downstream of the bow shock, the plasma and the electromagnetic disturbances of the shocked solar wind reach the ionosphere directly through the cusp and continuously carry a massive amount of energy to the ionosphere (Heikkila and Winningham, 1971; Yamauchi and Lundin, 2001). This localized energy generates strong electromagnetic wave activities in and above the iono- sphere, which results in wave-related non-thermal heating (e.g., Moore et al., 1999). This region is one of the largest sources of non-thermally escaping ions, which is summarized in Table 2. Note that solar wind also penetrates across the magnetopause outside the cusps at localized sites and in a non-steady manner (Sibeck et al., 1999; Yamauchi et al., 2003; Lundin et al., 2003; Fujimoto et al., 2006), and once the magnetosphere has been penetrated, solar wind has easy access to the ionosphere along the magnetic field, as observations have confirmed (Andersson et al., 2002; Yamauchi et al., 2003). Thus, the shielding boundary for a magnetized planet is not very solid, particularly for disturbed solar wind (solar wind is already disturbed when it passes through the bow shock).

If the planetary magnetic field is not strong enough to block solar wind, the planet is defined as unmagnetized (Fig. 1b). In this case, solar wind is blocked by the ionospheric plasma pressure as long as the planet has a substantial ionosphere (e.g., Zhang et al., 1991; Brace and Kliore, 1991; Luhmann and Bauer, 1992, Wahlund et al., 2005). This blockage is still achieved in the collision-free regime because the magnetic field cannot quickly diffuse into a good conductor (the ionosphere has high conductance), and it "piles up" in front of the conductor due to the electric induction current in the ionosphere (e.g., Alfvén and Fälthammar, 1963; Russell et al., 2006). In the dayside part, this magnetic piled-up region is called the ionopause. The piled-up IMF prevents both solar wind and ionospheric plasmas from crossing the ionopause by gyrotrapping, and as a result, SWDP and ionospheric plasma pressure are balanced by the magnetic pressure of the piled-up IMF, respectively. This picture has been confirmed by observations of both Venus and Mars (e.g., Zhang et al., 1990; Zhang et al., 1991; Russell et al., 2006). Because of this magnetic pile-up mechanism, the total magnetic field that confines the ionosphere and shields it from solar wind intrusion is the same regardless of how weak the IMF is (Luhmann et al., 1987).

While the interaction between the solar wind and the ionosphere is in the collision-free regime, it still causes strong losses of ionospheric ions due to large-scale momentum transfer (Wolff et al., 1980; Ong et al., 1991; Brace and Kliore, 1991; Lundin and Barabash, 2004; Carlsson et al., 2006). Furthermore, this shielding boundary (the 
ionopause) is not very solid (less solid than the magnetopause) because the IMF direction is not stable and the solar wind is disturbed downstream of the bow shock. Therefore, localized energy deposition through the ionosphere is more frequent than it is through the magnetopause. Such localized energy deposition is favorable for non-thermal heating.

If the ionospheric ion density is very low (due to, e.g., low EVU/FUV), the question arises whether direct collisions between the solar wind and the neutral atmosphere determine the boundary of solar wind access as illustrated in Fig. 1c. This is equivalent to the case where there is no IMF at all or the neutral atmosphere is much thicker than the ionosphere. However, the very high ionizing EUV/FUV flux predicted for the early Sun produces a dense ionosphere above the collisional neutral atmosphere of the Earth-type planet ( $c f$. section 5 below and textbook by Rees, 1989). Furthermore, even Titan, which receives very low levels of EUV/FUV, has a thick ionosphere (section 2.1) such that the ionospheric pressure balances with Saturn's corotation wind in the collision-free regime through the piled-up magnetic field. Generally, neutrals that are exposed to solar wind are expected to be quickly lost through the ion pickup process once they are ionized inside the solar wind, which causes a fast erosion of the neutral atmosphere until the ionospheric pressure dominates in balancing with the SWDP. Therefore, we do not take into consideration the situation illustrated in Fig. 1c. Note that we do not exclude the possibility of an extended hydrogen exosphere (Luhmann and Bauer, 1992; Kulikov et al., 2006) because the proton gyroradius in the piled-up IMF is normally shorter than the mean free pass of neutral-proton collision.

\section{SOLAR WIND DEPENDENCE}

For both magnetized and unmagnetized planets, IMF does not affect significantly the amount of neutral escape [(1)-(3) in Table 1] because the exosphere is not much affected by the IMF. A high SWDP generally increases the charge-exchange ionization and the electron impact ionization of the exosphere inside the solar wind, but not below the ionopause/magnetopause where the exobase is located. Therefore, SWDP does not much affect Jeans escape or photo- chemical escape, and we consider only non-thermal ion escape mechanisms [(4)-(6) in Table 1] in this section.

\subsection{Magnetized planet (Earth)}

For a magnetized planet with a strong magnetic field such as the present Earth, the ionosphere is always well below the magnetopause, as illustrated in Fig. 1a, and the pickup loss is expected to be very small compared to other nonthermal ion losses by, for example, large-scale momentum transfer and non-thermal (wave-related) heatings. For these non-thermal outflows, past terrestrial observations over 30 years have revealed some of the solar input dependencies as summarized in section 2.2 (see Table 2) and below.

An increase in SWDP or IMF variability causes a non-linear increase in non-thermal heating of ionospheric ions by more than an order of magnitude within several hours (Moore et al., 1999; Cully et al., 2003). The change is largest in the dayside polar region near the cusp ( $c f$. Fig. 1a), and the related escaping ions enhance the mass loading effect and subsequent large-scale momentum transfer (Yamauchi and Lundin, 1997, 2001). The SWDP also affects the magnetopause (dashed line in Fig. 1a). A high SWDP moves the magnetopause location toward Earth through compression/erosion (e.g., Akasofu, 1977) and deforms the magnetopause shape, through instabilities, to wavy rather than laminar (e.g., Sibeck et al., 1999; Yamauchi et al., 2003; Fujimoto et al., 2006 ).

An increase in IMF strength also causes magnetopause shrinkage/erosion (particularly for specific IMF directions (e.g., Akasofu, 1977), some increase in non-thermal heating (Cully et al., 2003; Arvelius, 2005), and an increase in large-scale momentum transfer through large-scale plasma instabilities (e.g., Fujimoto et al., 2006). The magnetopause shrinkage/erosion is often attributed to the dayside reconnection process, which transfers the mass from the dayside to the nightside (e.g., Hones et al., 1974). Although this is one of the largest mass transfer processes, the majority of the transferred mass related to the reconnection process is believed to return back to the dayside because the total magnetic flux of the terrestrial magnetic field is conserved, which is not the case for other escape processes (Yamauchi et al., 2005b). Therefore, the net escape of ions by re- 
connection and other large-scale processes is completely unknown. Poor knowledge of the return flow is not limited to the reconnection process. We do not even know the returning amount of outflow ions for other mechanisms such as wave-related heating. Yet we can expect that the increase in local escape most likely leads to an increase in total escape flux.

\subsection{Unmagnetized planets (Mars, Venus, the moon Titan)}

We next consider an unmagnetized planet, as illustrated in Fig. $1 \mathrm{~b}$ and Fig. 2. The relative location of the shielding boundary (dashed line) and the exosphere in the case of the unmagnatized planet is more important than it is in the magnetized case because the picked-up loss involves neutrals beyond the ionopause. For the other non-thermal ion escape mechanisms, we employ terrestrial observations to supplement the relatively insufficiently investigated solar wind dependence of the ionospheric condition and escape (e.g., Zhang et al., 1990, 1991; Ong et al., 1991).

As mentioned in section 3, the IMF strength does not change the total piled-up magnetic field around the ionopause. Terrestrial observations indicate that IMF strength influences non-thermal heatings much less than the other parameters do (Arvelius, 2005). On the contrary, an increase in the SWDP means that the pressure balance altitude decreases toward the planet as shown in Fig. 2a (Zhang et al., 1991), which results in more neutrals existing beyond the ionopause. Together with the increase in the ionization rate (by chargeexchange between ions and neutrals and by electron impact ionization), an increase in the SWDP should increase the pickup loss by solar wind. For the other non-thermal ion escape mechanisms [(5) and (6) in Table 1], activation of the escape process by the localized solar wind energy deposit into the ionosphere is analogous to the activation at a magnetized planet, and we expect that an increase in the SWDP activates the largescale momentum transfer in the boundary region and the non-thermal ion heating in the ionosphere (section 4.1). On the other hand, Pioneer Venus Orbiter's cold plasma instrument found a decrease of nightside ionotail density when SWDP increased (Brace et al., 1990). Since the density and characteristic energy are normally anti-correlated and the density does not necessarily represent flux (Arvelius, 2005), one cannot presently conclude anything without hot plasma measurements. Results from Mars Express/ (a) SWDP dependence
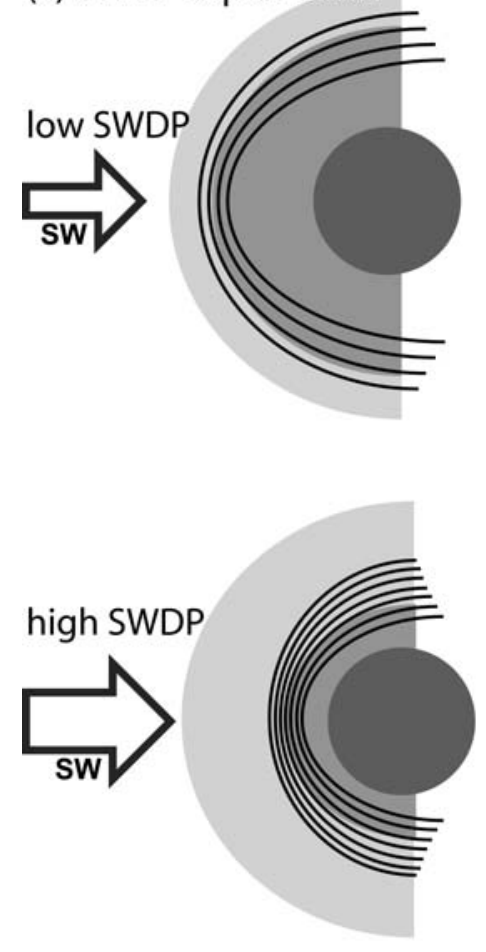

(b) UV dependence
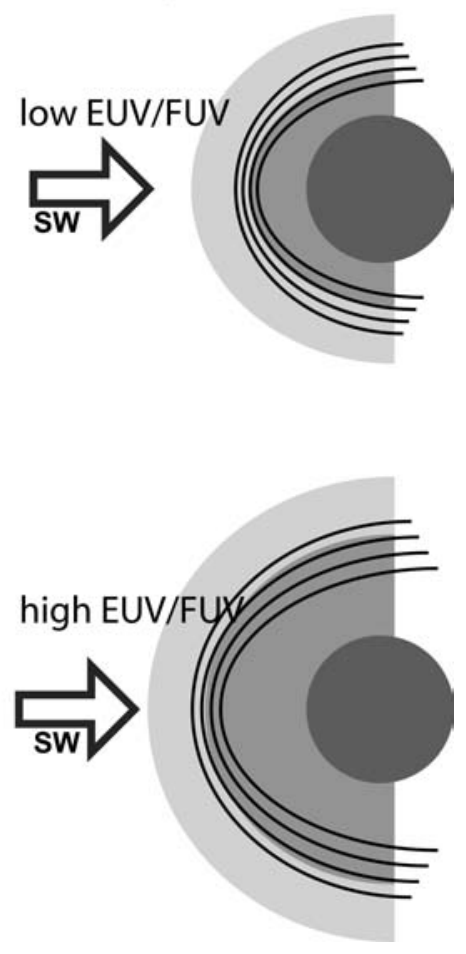

FIG. 2. Illustration of the interaction between the solar wind and an unmagnetized planet (same as Fig. 1b) for different solar parameters: (a) high and low SWDP and (b) high and low EUV/FUV flux. The ionopause location depends on both SWDP (due to compression) and EUV/FUV (due to enhanced ionization), while the exosphere depends on EUV / FUV but not on SWDP. Note that a high SWDP also intensifies the piled-up magnetic field because its magnetic pressure must stop the solar wind. 
Venus Express for the SWDP dependence will shed light on these issues.

Variability in the IMF direction means that the new IMF may weaken the piled-up magnetic field. For example, a $180^{\circ}$ change in the IMF direction means a cancellation of the piled-up old IMF by a new IMF at the ionopause and causes the ions that are trapped in the ionopause to be exposed to solar wind and subsequently be picked up or released (Ong et al., 1991). The cancellation of the piled-up IMF also means that the magnetic pressure is no longer strong enough to sustain the SWDP; that is, until a new magnetic configuration is set up above the ionosphere. Together with the loss of ions and weakening of the magnetic shielding, the pressure balance point (the ionopause) is expected to move dynamically toward the planet. Variations in the IMF direction, therefore, cause variations in the ionopause height, which triggers large-scale instabilities and thus enhances the momentum transfer as well as the localized solar wind penetration into a lower altitude than a stationary IMF case. In fact, Ong et al. (1991) found an increase of observing frequency of detached ion cloud from Venus when the IMF direction changes from Pioneer Venus Orbiter (PVO).

The most relevant terrestrial counterpart is probably the cusp region because the magnetic obstacle is very weak there; observations at the cusp region show that the solar wind and its energy access much more easily during higher SWDP and more variable IMF (e.g., Moore et al., 1999; Yamauchi and Lundin, 2001). In summary, a variable IMF (and probably a high SWDP) causes an increase in non-thermal energization (e.g., by electromagnetic wave activity that corresponds to (5) in Table 1) and related ion losses of the ionospheric ions. It is also favorable for largescale momentum transfer near the ionopause.

\section{SOLAR EUV/FUV DEPENDENCE}

We next consider the EUV/FUV dependence. In this section, we simply call them UV. The most direct UV effect is on ionospheric and exospheric height.

According to the simple Chapman model in which one-component atmosphere is assumed with scale height $H$ (counter proportional to the gravity and mass of the atom/ion), cross section for ionization $a$, incoming solar flux $F_{0}$, and den- sity $n_{0}$ at altitude $z=0$, the peak altitude $z_{\max }$ and peak production rate $q_{\max }$ are given as

$$
\begin{gathered}
z_{\max }\left(\theta, F_{0}, H\right)=H \ln \left(n_{0} a H / \cos (\theta)\right) \\
q_{\max }\left(\theta, F_{0}, H\right)=F_{0} \cos (\theta) /\left(2.7^{*} H\right)
\end{gathered}
$$

where $\theta$ is the solar zenith angle. The peak altitude does not depend on $F_{0}$, but on $H$ (i.e., gravity), whereas the peak production rate depends on both $F_{0}$ and $H$. The final profile is significantly modified by the transport of ions (vertical motion due to heating, which is proportional to q), and it moves the peak for $n(z)$ to a higher altitude with a less sharp $n(z)$ profile than the production profile (e.g., textbook by Rees, 1989). As a result, the ionospheric extent depends on both $F_{0}$ (solar UV) and gravity (the size of the planet). A higher UV flux or a smaller planet (i.e., gravity) leads to a higher ionopause altitude. In fact, observations of Earth, Mars, and Venus have revealed a large variation of the ionospheric density and its extent (or ionopause height) between solar maximum and solar minimum (Evans, 1977; Zhang et al., 1990; Kliore and Luhmann, 1991).

When the neutral atmosphere (exosphere) is heated to expand for high UV flux (e.g., Kulikov et al., 2006), such a condition means a higher ionization rate, e.g., by UV, electron impact, and the CIV mechanisms (section 2.1). All terrestrial planets with substantial atmospheres (Earth, Venus, Mars, and the moon Titan) have an ionosphere that is expanded to above the exobase, regardless of solar wind and UV conditions. Therefore, we assume in our qualitative assessment that the ionopause is always located above the exobase. Figure $2 b$ illustrates the resultant UV dependence of the relative location of the exosphere and the ionopause.

This assumption directly indicates fewer neutrals above the ionopause than is the case for existing models (e.g., Kulikov et al., 2007); hence, less pickup loss, because newly born ions below the ionopause/magnetopause are protected from solar wind and IMF and trapped within the planetary magnetosphere/ionosphere as soon as they are ionized. For the same reason, the thermal escape rate of neutrals below the ionopause (eventually valid for the magnetopause, too) is also expected to be lower than it is for existing models because a substantial portion of neutrals beyond the escape velocity are ionized by the extra ionization such as CIV or extra electron impact ion- 
ization as discussed in section 2.1. Therefore, a high UV flux might not cause as high a thermal escape rate as many models predict.

\subsection{Magnetized planet (Earth)}

At magnetized planets, the intensity of the UV flux does not generally affect the magnetopause location (dashed line in Fig. 1a), which is determined by the planetary intrinsic magnetic field and the SWDP. The UV increase certainly increases the exospheric height, but for the reason mentioned above, its effect on the thermal escape rate is expected to be smaller than predictions by models, unless the exosphere extends far above the magnetopause. For such an extended exosphere, the ionization rate must also be high, such that the entire setup becomes similar to that of the unmagnetized planets, which will be discussed in the next subsection.

However, a high UV flux causes a higher, hotter, and denser ionosphere and enhances nonthermal heating and related ion escape as mentioned in section 2.2 (Moore et al., 1999; Cully et al., 2003; Ebihara et al., 2006; and references therein). By extrapolating the result by Cully et al. (2003), an increase of $F_{10.7}$ flux by an order of magnitude (corresponding to the early Sun) might cause an increase of non-thermal ion escape from the ionosphere by anywhere from 4 orders of magnitude (power law extrapolation) to 9 orders of magnitude (exponential extrapolation) for $\mathrm{O}^{+}$and by $1 \sim 2$ orders of magnitude for $\mathrm{H}^{+}$. The $\mathrm{O}^{+}$escape flux depends on the solar activity and geomagnetic activity much more than the $\mathrm{H}^{+}$flux does.

The ion pickup loss of exospheric origin is not much affected by the UV flux change because the number of neutrals outside the magnetopause is not very large as long as the ionosphere and exobase are far below the magnetopause (this is the definition of magnetized planets in this paper). The other form of pickup, i.e., direct pickup of ions which have crossed the magnetopause from the magnetosphere, is mostly already counted in the non-thermal heating because most of these ions are already energized to more than the escape velocity before they reach the magnetopause (Arvelius et al., 2005) and do not need the assistance of the pickup process for their escape. On the other hand, the subsequent mass loading by these ions enhances large-scale momentum transfer (Yamauchi and Lundin, 1997, 2001) and contributes further UV dependence. Finally, the UV dependence of other forms of momentum transfer, such as reconnection-related large-scale mass release from the tail, is not well studied yet. Possible ionospheric factors that may affect reconnection-related escape include increase in the ionospheric conductance and change in the composition at the reconnection site.

\subsection{Unmagnetized planets (Mars, Venus, the moon Titan)}

Again, we largely rely on terrestrial observations and a synthesis of observations for Venus, Mars, and Titan as summarized in section 2.2 as well as observed solar UV dependencies at Venus (e.g., Moore et al., 1990; Brace et al., 1990; Zhang et al., 1990; Zhang et al., 1991). As described in the beginning of this section, thermal escape and the pickup of newly ionized neutrals by solar wind are not as strongly UV dependent as existing models have predicted. As illustrated in Fig. 2b, a high UV flux causes a hot expanded exosphere, which is favorable to enhanced thermal escape, while the expanded ionosphere reduces this contribution by the extra ionization of these neutrals with escaping velocity inside the ionosphere. The reduction of escape by the expanded ionosphere has more to do with ion pickup loss than with thermal escape because only those neutrals above the ionopause can contribute to the pickup process, while a substantial portion of neutrals inside the ionosphere may escape by thermal escape. It might also be possible that the expansion of the ionosphere could be more UV dependent than the expansion of the exosphere, which would cause a reduction of the total number of exospheric neutrals above the ionopause for high UV. In this case, the ion pickup loss would decrease with increasing UV.

For non-thermal ion escape mechanisms, we note that a higher UV flux causes a hotter and more expanded ionosphere and a larger solar wind-ionosphere interaction region (i.e., ionopause). While a larger solar wind-ionosphere interaction region means higher large-scale momentum transfer, a hotter and more expanded ionosphere means a stronger non-thermal heating because of a larger amount of the localized energy deposition (see section 3), which, together with the higher temperature, enhances the localized electromagnetic wave activity in the ionosphere, as suggested by terrestrial observations 
Table 3. Major Non-Thermal Ion Escape Mechanisms from Magnetized Planets

\begin{tabular}{llllll}
\hline Increase in & \multicolumn{1}{c}{$\begin{array}{c}\text { Pickup } \\
\text { small })\end{array}$} & $\begin{array}{c}\text { Large-scale } \\
\text { momentum } \\
\text { transfer }\end{array}$ & $\begin{array}{c}\text { Non-thermal } \\
\text { heating of ions }\end{array}$ & $\begin{array}{c}\text { Cf. Jeans }+ \\
\text { photochemical }\end{array}$ & $\begin{array}{c}\text { O/H ratio } \\
\text { of escape }\end{array}$ \\
\hline EUV/FUV flux & No change & a & Large increase & Increase & b \\
SWDP & No change & Increase & Large increase & No change & Large increase \\
IMF intensity & No change & a & Increase & No change & Increase \\
IMF variability & No change & a & Increase & No change & Increase \\
Gravity & Some decrease & No change & $?$ & Some decrease & Increase \\
\hline
\end{tabular}

activity itself increases but the amount of loss is an open issue.

${ }^{b}$ Increase or decrease depending on the relative importance of non-thermal heating.

of non-thermal ion escape (Cully et al., 2003). In fact, PVO observation of solar cycle dependence based on cold plasma data shows an increase of total non-thermal ion escape [(4)-(6) in Table 1] during solar maximum (e.g., Moore et al., 1990; Brace et al., 1990). The Mars Express and Venus Express missions are expected to reveal the hot plasma part of non-thermal ion escape over the solar cycle.

Finally, we mention the gravity effect. In Equations 1 and 2, the dependence on gravity appears to be slightly less than the logarithmic response. This logarithmic response is valid for both the exosphere and the ionosphere; that is, the exosphere shrinks more than the ionosphere in actual distance (in $\mathrm{km}$ ) for higher gravity. Furthermore, gravity effect on the ionopause height is reduced by compression by the SWDP, while gravity effect on the exospheric extent is not much affected by the SWDP (such a difference does not exist for a magnetized planet). Therefore, for an unmagnetized planet, higher gravity causes more shrinkage of the neutral atmosphere than of the ionosphere, and lower gravity causes more expansion. (Note that the ionopause is still expected to be located higher than the exobase). Recent Cassini observations have confirmed more massive escape of cold ions from Titan than from Venus (Wahlund et al., 2005, 2006). This is consistent with the above argument for both the UV dependence and the gravity dependence.

\section{SUMMARY}

We have discussed how the ionosphere may play an important role in controlling the atmospheric loss at early Earth and Earth-type planets and have qualitatively evaluated its positive/ negative effects as a function of the solar/stellar wind and radiation. The ionosphere contributes directly to atmospheric loss processes: (1) It is a source of non-thermal ion escape by, for example, non-thermal ion heating for both magnetized and unmagnetized planets. The relevant escape mechanisms are more solar wind/radiation dependent than thermal escape, particularly for $\mathrm{O}^{+}$compared to $\mathrm{H}^{+}$, which is important in determining the oxidized state of the atmosphere. (2) It is a protector from a solar wind-driven escape of ions by gyro-

Table 4. Major Non-Thermal Ion Escape Mechanisms from Unmagnetized Planets

\begin{tabular}{|c|c|c|c|c|c|}
\hline Increase in & Pickup & $\begin{array}{c}\text { Large-scale } \\
\text { momentum } \\
\text { transfer }\end{array}$ & $\begin{array}{l}\text { Non-thermal } \\
\text { heating of ions }\end{array}$ & $\begin{array}{l}\text { Cf. Jeans + } \\
\text { photochemical }\end{array}$ & $\begin{array}{l}\mathrm{O} / \mathrm{H} \text { ratio } \\
\text { of escape }\end{array}$ \\
\hline EUV/FUV flux & Decrease?a & Increase & Increase & Increase & $\mathrm{b}$ \\
\hline SWDP & Increase & c & c & No change & Large increase \\
\hline IMF intensity & No change & No change & No change & No change & No change \\
\hline IMF variability & Some increase & Large increase & Increase & No change & Increase \\
\hline Gravity & Decrease & No change & $?$ & Decrease & Increase \\
\hline
\end{tabular}

aNot clear. The comparison between Titan and Mars suggests decrease, but this simple comparison might not appropriate to model the ancient escape.

${ }^{b}$ Increase or decrease depending on the relative importance of non-thermal heating.

${ }^{\mathrm{c}}$ Although PVO statistics shows decrease in cold plasma density, we may expect an increase of escape flux based on terrestrial results (see section 4.2). 
trapping to the piled-up IMF for unmagnetized planets. Therefore, the ionopause location relative to the exospheric extent is a controlling factor for the ion-pickup loss. For a magnetized planet, the pickup loss of the exospheric neutrals is very little because the exosphere does not extend much above the magnetopause. (3) It also partly contributes to large-scale momentum transfer and resultant largescale ion escape. As a result, the ionosphere makes the solar parameter dependencies of the atmospheric loss very dynamic for both thermal and nonthermal escape mechanisms.

Tables 3 and 4 summarize the expected solar parameter dependencies of ion escape for magnetized and unmagnetized planets, respectively. A high atmospheric loss rate is expected when the planetary size is small, SWDP is high, IMF is variable, and the EUV/FUV flux is high. However, the dependence is different between thermal escape (small change) and non-thermal escape (large change). In our assessment, it may even be possible that the EUV/FUV dependence on the pickup loss is negative because of the expansion of the ionopause that keeps plasma within the ionosphere. Therefore, non-thermal escape from the ionosphere is as important as thermal escape or ion pickup from the exosphere of ancient Earth and Venus, when the solar EUV/FUV flux was very high (and solar wind was possibly very active). For Mars, on the other hand, the escape velocity of hydrogen is much lower than the CIV due to the low gravity; hence, unless the planet is strongly magnetized, thermal escape is expected to be the most important process, even 4-4.5 Gyr ago.

Since the $\mathrm{O} / \mathrm{H}$ ratio of the observed non-thermal escape depends non-linearly on the solar parameters with a high $\mathrm{O} / \mathrm{H}$ ratio for a high $\mathrm{EUV} / \mathrm{FUV}$, SWDP, IMF, or a variable IMF, the ancient Earth and Venus must have had a much higher $\mathrm{O} / \mathrm{H}$ ratio of atmospheric escape than has been predicted by existing models. The higher $\mathrm{O} / \mathrm{H}$ ratio of escape is important in modeling the oxidation state of the ancient atmosphere. Thus, to understand the atmospheric conditions at the time of the emergence of life and its early evolution, we need proper modeling of the ancient ionosphere along with proper assumptions of the static and dynamic conditions of the solar input and the ionosphere.

In this study, we did not consider many internal effects. The conditions of the upper neutral atmosphere, such as composition, temperature, and convection, cause large uncertainty in the ionopause location (for the unmagnetized case) and non-thermal heating (for the magnetized case). Volcanic activity should also significantly affect solar wind interaction because ancient eruptions, which were much stronger than at present, may have easily caused different $\mathrm{O} / \mathrm{H}$ ratios in the escaping flux into the solar wind, as indicated by Io's volcano. Direct ion pickup of volcanic material may affect the atmospheric $\mathrm{pH}$.

\section{ACKNOWLEDGMENTS}

This work is partly supported by the Swedish Research Council (Vetenskaprådet) \#621-20055005. M. Yamauchi wishes to thank programs for the disabled in Sweden, which have made it possible for him to work.

\section{ABBREVIATIONS}

CIV, critical ionization velocity; EUV, extreme ultraviolet; FUV, far ultraviolet; IMF, interplanetary magnetic field; PVO, Pioneer Venus Orbiter; SW, solar wind; SWDP, solar wind dynamic pressure.

\section{REFERENCES}

Abe, Y., Ohtani, E., Okuchi, T., Righter, K., and Drake, M. (2000) Water in the early Earth. In Origin of the Earth and Moon, edited by R.M. Canup and K. Righter, University of Arizona Press, Tucson, pp. 413-433.

Ågren, K., Wahlund, J.-E., Modolo, R., Lummerzheim, D., Galand, M., Muller-Wodarg, I., Canu, P., Kurth, W.S., Cravens, T., Yelle, R., Waite, J.H., Jr., Coates, A., Lewis, G., Young, D., Bertucci, C., and Doughterty, M.K. (2007) On magnetospheric electron impact ionization and dynamics in Titan's ram-side and polar ionosphere-a Cassini case study, submitted to Annales Geophysicae.

Akasofu, S.-I. (1977) Physics of Magnetospheric Substorms, Astrophysics and Space Science Library, Vol. 47, Reidel, Dordrecht, Holland.

Alfvén, H. and Fälthammar, C.G. (1963) Cosmical Electrodynamics, Fundamental Principles, Clarendon, Oxford.

Andersson, L., Wahlund, J.-E., Clemmons, J., Gustavsson, B., and Eliasson, L. (2002) Electromagnetic waves and bursty electron acceleration: implications from Freja. Annales Geophysicae 20, 139-150.

Arvelius, S. (2005) Energization and acceleration of dayside polar outflowing oxygen, PhD thesis. IRF Scientific Report 287, Swedish Institute of Space Physics (IRF), Kiruna, Sweden. 
Arvelius, S., Yamauchi, M., Nilsson, H., Lundin, R., Hobara, Y., RËme, H., Bavassano-Cattaneo, M.B., Paschmann, G., Korth, A., Kistler, L.M., and Parks, G.K. (2005) Statistics of high-altitude and high-latitude $\mathrm{O}^{+}$ ion outflows observed by Cluster/CIS. Annales Geophysicae 23, 1909-1916.

Ayres, T.A. (1997) Evolution of the solar ionizing flux. J. Geophys. Res. 102(E1), 1641-1651.

Barabash, S., Fedorov, A., Lundin, R., and Sauvaud, J.-A. (2007) Measurements from Mars Express show that the solar wind is removing only a small amount of Mars' atmosphere, implying that the formally abundant $\mathrm{H}_{2} \mathrm{O}$ and $\mathrm{CO}_{2}$ remain underground. Science 315, 501-503.

Brace, L.H., and Kliore, A.J. (1991) The structure of the Venus ionosphere. Space Sci. Rev. 55, 81-163.

Brace, L.H., Theis, R.F., and Mihalov, J.D. (1990) Response of nightside ionosphere and ionotail of Venus to variations in solar EUV and solar wind dynamic pressure. J. Geophys. Res. 95, 4075-4084.

Brecht, S.H., and Ledvina, S.A. (2006) The solar wind interaction with the martian ionosphere/atmosphere. Space Sci. Rev. 126, 15-38.

Carlsson, E., Fedorov, A., Barabash, S., Budnik, E., Grigoriev, A., Gunell, H., Nilsson, H., Sauvaud, J.-A., Lundin, R., Futaana, Y., Holmström, M., Andersson, H., Yamauchi, M., Winningham, J.D., Frahm, R.A., Sharber, J.R., Scherrer, J., Coates, A.J., Linder, D.R., Kataria, D.O., Kallio, E., Koskinen, H., Säles, T., Riihelä, P., Schmidt, W., Kozyra, J., Luhmann, J., Roelof, E., Williams, D., Livi, S., Curtis, C.C., Hsieh, K.C., Sandel, B.R., Grande, M., Carter, M., Thocaven, J.-J., McKennaLawlor, S., Orsini, S., Cerulli-Irelli, R., Maggi, M., Wurz, P., Bochsler, P., Krupp, N., Woch, J., Fränz, M., Asamura, K., and Dierker, C. (2006) Mass composition of the escaping plasma at Mars. Icarus 182, 320-328.

Chappell, C.R., Olsen, R.C., Green, J.L., Johnson, J.F.E., and Waite, J.H., Jr. (1982) The discovery of nitrogen ions in the Earth's magnetosphere. Geophys. Res. Lett. 9, 937-940.

Commeyras, A., Boiteau, L., Vandenabeele-Trambouze, O., and Selsis, F. (2006) Peptide emergence, evolution and selection on the primitive Earth. II. The primary pump scenario. In Lectures in Astrobiology, edited by M. Gargaud, B. Barbier, H. Martin, and J. Reisse, Vol. 1, Part 2, Springer, New York, pp. 147-170.

Cravens, T.E., Robertson, I.P., Clark, J., Wahlund, J.-E., Waite, J.H., Jr., Ledvina, S.A., Niemann, H.B., Yelle, R.V., Kasprzak, W.T., Luhmann, J.G., McNutt, R.L., Ip, W.-H., De La Haye, V., Muller-Wodarg, I., Young, D.T., and Coates, A.J. (2005) Titan's ionosphere: model comparisons with Cassini Ta data. Geophys. Res. Lett. 32, L12108.

Cully, C.M., Donovan, E.F., Yau, A.W., and Arkos, G.G. (2003) Akebono/Suprathermal Mass Spectrometer observations of low-energy ion outflow: dependence on magnetic activity and solar wind conditions. J. Geophys. Res. 108, 1093.

Ebihara, Y., Yamada, M., Watanabe, S., and Ejiri, M. (2006) Fate of outflowing suprathermal oxygen ions that originate in the polar ionosphere. J. Geophys. Res. 111, A04219.
Eklund, U., Lundin, R., and Sandahl, I. (1997) Measurements of $\mathrm{O}^{+}$in the high latitude magnetosheath. Physics and Chemistry of the Earth 22, 639-644.

Evans, J.V. (1977) Satellite beacon contributions to studies of the structure of the ionosphere. Rev. Geophys. 15, 325-350.

Fujimoto, M., Nakamura, T.K.M., and Hasegawa, H. (2006) Cross-scale coupling within rolled-up MHDscale vortices and its effect on large-scale plasma mixing across the magnetospheric boundary. Space Sci. Rev. 122, 3-18.

Gordiets, B.F., Kulikov, Yu.N., Markov, M.N., and Morav, M.Ya. (1982) Numerical modelling of the thermospheric heat budget. J. Geophys. Res. 87, 4504-4514.

Hamilton, D.C., Gloeckler, G., Ipavich, F.M., Studemann, W., Wilken, B., and Kremser, G. (1988) Ring current development during the great geomagnetic storm of February 1986. J. Geophys. Res. 93, 14343-14355.

Hashimoto G.L., Abe, Y., and Sugita, S. (2007) The chemical composition of the early terrestrial atmosphere: formation of a reducing atmosphere from CI-like material. J. Geophys. Res. 112, E05010.

Heikkila, W.J. and Winningham, J.D. (1971) Penetration of magnetosheath plasma to low altitudes through the dayside magnetospheric cusps. J. Geophys. Res. 76, 883-891.

Hones. E.W., Jr., Lui, A.T.Y., Bame, S.J., and Singer, S. (1974) Prolonged tailward flow of plasma in the thinned plasma sheet observed at $\mathrm{r} \sim 18 \mathrm{RE}$ during substorms. J. Geophys. Res. 79, 1385-1392.

Kasting, J.F. and Brown, L.L (1998) The early atmosphere as a source of biogenic compounds. In The Molecular Origin of Life, edited by A. Brack, Cambridge University Press, Cambridge, pp. 35-56.

Kasting, J.F. and Catling, D. (2003) Evolution of a habitable planet. Annu. Rev. Astron. Astrophys. 41, 429-463.

Kliore, A.J., and Luhmann, J.G. (1991) Solar cycle effects on the structure of the electron density profiles in the dayside ionosphere of Venus. J. Geophys. Res. 96, 21281-21289.

Kondo, T., Whalen, B.A., Yau, A.W., and Peterson, W.K. (1990) Statistical analysis of upflowing ion beam and conic distributions at DE 1 altitudes. J. Geophys. Res. 95, 12091-12102.

Kulikov, Yu.N., Lammer, H., Lichtenegger, H.I.M., Terada, N., Ribas, I., Kolb, C., Langmayr, D., Lundin, R., Guinan, E.F., Barabash, S., and Biernat, H.K. (2006) Atmospheric and water loss from early Venus. Planet. Space Sci. 54, 1425-1444.

Kulikov, Yu.N., Lammer, H., Lichtenegger, H.I.M., Penz, T., Breuer, D., Spohn, T., Lundin, R., and Biernat, H.K. (2007) A comparative study of the influence of the active young Sun on the early atmospheres of Earth, Venus, and Mars. Space Sci. Rev. 129, 207-243.

Lammer, H., Lichtenegger, H.I.M., Kolb, C., Ribas, I., Guinan, E.F., Abart, R., and Bauer, S.J. (2003) Loss of water from Mars: implications for the oxidation of the soil. Icarus 165, 9-25.

Lammer, H., Lichtenegger, H.I.M., Penz, T., Amerstorfer, U.V., Kolb, C., and Biernat, H.K. (2005a) Solar wind ero- 
sion of the martian and venusian atmospheres. In Solar-Planetary Relations, edited by H.K. Biernat, H. Lammer, D.F. Vogl, and S. Mühlbachler, Research Signpost, Trivandrum, India, pp. 209-217.

Lammer, H., Selsis, F., Penz, T., Amerstorfer, U.V., Lichtenegger, H.I.M., Kolb, C., and Ribas, I. (2005b) Atmospheric evolution and the history of water on Mars. In Water on Mars and Life, edited by T. Tokano, SpringerVerlag, Berlin, pp. 25-43.

Lammer, H., Lichtenegger, H.I.M., Kulikov, Y.N., Grießmeier, J.-M., Terada, N., Erkaev, N.V., Biernat, H.K., Khodachenko, M.L., Ribas, I., Penz, T., and Selsis, F. (2007) Coronal mass ejection (CME) activity of low mass $M$ stars as an important factor for the habitability of terrestrial exoplanets. II. CME-induced ion pick up of Earth-like exoplanets in close-in habitable zones. Astrobiology 7, 185-207.

Lassen, K. and Friis-Christensen, E. (1995) Variability of the solar cycle length during the past five centuries and the apparent association with terrestrial climate. J. Atmos. Terr. Phys. 57, 835-845.

Luhmann, J.G. and Bauer, S.J. (1992) Solar wind effects on atmosphere evolution at Venus and Mars. In Venus and Mars: Atmospheres, Ionospheres, and Solar Wind Interactions, edited by J.G. Luhmann, M. Tatrallyay, and R.O. Pepin, American Geophysical Union, Washington, DC, pp. 417-430.

Luhmann, J.G., Russell, C.T., Scarf, F.L., Brace, L.H., and Knudsen, W.C. (1987) Characteristics of the Marslike limit of the Venus-solar wind interaction J. Geophys. Res. 92, 8545-8557.

Luhmann, J.G., Johnson, R., and Zhang, M.G.H. (1992) Evolutionary impact of sputtering of the martian atmosphere by $\mathrm{O}^{+}$pick up ions. Geophys. Res. Lett. 19, 2151-2154.

Lundin, R. and Barabash, S. (2004) Evolution of the martian atmosphere and hydrosphere: solar wind erosion studied by ASPERA-3 on Mars Express. Planet. Space Sci. 52, 1059-1071.

Lundin, R. and Dubinin, E.M. (1992) Phobos-2 results on the ionospheric plasma escape from Mars. Adv. Space Res. 12, 255-263.

Lundin, R., Zakharov, A., Pellinen, R., Barabasj, S.W., Borg, H., Dubinin, E.M., Hultqvist, B., Koskinen, H., Liede, I., and Pissarenko, N. (1990) ASPER/PHOBOS measurements of the ion outflow from the martian ionosphere. Geophys. Res. Lett. 17, 873-876.

Lundin, R., Dubinin, E.M., Barabash, S.V., Koskinen, H., Norberg, O., Pissarenko, N., and Zakharov, A.V. (1991) On the momentum transfer of the solar wind to the martian topside ionosphere. Geophys. Res. Lett. 18, 1059-1062.

Lundin, R., Sauvaud, J.-A. Rème, H., Balogh, A., Dandouras, I., Bosqued, J.M., Carlson, C., Parks, G.K., Möbius, E., Kistler, L.M., Klecker, B., Amata, E., Formisano, V., Dunlop, M., Eliasson, L., Korth, A., Lavraud, B., and McCarthy, M. (2003) Evidence for impulsive solar wind plasma penetration through the dayside magnetopause. Annales Geophysicae 21, 457-472.
Lundin, R., Barabash, S., Andersson, H., Holmström, M., Grigoriev, A., Yamauchi, M., Sauvaud, J.-A., Fedorov, A., Budnik, E., Thocaven, J.-J., Winningham, D., Frahm, R., Scherrer, J., Sharber, J., Asamura, K., Hayakawa, H., Coates, A., Linder, D.R., Curtis, C., Hsieh, K.C., Sandel, B.R., Grande, M., Carter, M., Reading, D.H., Koskinen, H., Kallio, E., Riihela, P., Schmidt, W., Säles, T., Kozyra, J., Krupp, N., Woch, J., Luhmann, J., McKenna-Lawler, S., Cerulli-Irelli, R., Orsini, S., Maggi, M., Mura, A., Milillo, A., Roelof, E., Williams, D., Livi, S., Brandt, P., Wurz, P., and Bochsler, P. (2004) Solar wind-induced atmospheric erosion on Mars: first results from ASPERA-3 on Mars Express. Science 305, 1933-1936.

Ma, Y., Nagy, A.F., Sokolov, I.V., and Hansen, K.C. (2004) Three-dimensional, multispecies, high spatial resolution MHD studies of the solar wind interaction with Mars. J. Geophys. Res. 109, A07211.

McFadden, J.P., Carlson, C.W., Strangeway, R., and Moebius, E. (2003) Observations of downgoing velocity dispersed $\mathrm{O}^{+}$and $\mathrm{H}^{+}$in the cusp during magnetic storms. Geophys. Res. Lett. 30, 1947-1951.

Moore, K.R., McComas, D.J., Russell, C.T., and Mihalov, J.D. (1990) A statistical study of ions and magnetic fields in the Venus magnetotail. J. Geophys. Res. 95, 12005-12018.

Moore, T.E., Lundin, R., Alcayde, D., Andre, M., Ganguli, S.B., Temerin, M., and Yau, A. (1999) Chapter 2-Source processes in the high-latitude ionosphere. Space Sci. Rev. 88, 7-84.

Norqvist, P., Andre, M., and Tyrland, M. (1998) A statistical study of ion energization mechanisms in the auroral region. J. Geophys. Res. 103, 23459-23474.

Ong, M., Luhmann, J.G., Russell, C.T., Strangeway, R.J., and Brace, L.H. (1991) Venus ionospheric "clouds:" relationship to the magnetosheath field geometry. J. Geophys. Res. 96, 11133-11144.

Owen, T.C. (1998) The origin of the atmosphere. In The Molecular Origin of Life, edited by A. Brack, Cambridge University Press, Cambridge, pp. 13-34.

Peratt, A.L. and Verschuur, G.L. (2000) Observation of the CIV effect in interstellar clouds: a speculation on the physical mechanism for their existence. IEEE Trans. Plasma Sci. 28, 2122-2127.

Pollack, J.B. (1991) Kuiper Prize lecture-Present and past climates of the terrestrial planets. Icarus 91, 173-198.

Rees, M.H. (1989) Physics and Chemistry of the Upper Atmosphere, Cambridge Atmospheric and Space Science Series, Cambridge University Press, Cambridge.

Rème, H., Aoustin, C., Bosqued, J.M., Dandouras, I., Lavraud, B., Sauvaud, J.A., Barthe, A., Bouyssou, J., Camus, Th., Coeur-Joly, O., Cros, A., Cuvilo, J., Ducay, F., Garbarowitz, Y., Medale, J.L., Penou, E., Perrier, H., Romefort, D., Rouzaud, J., Vallat, C., Alcaydé, D., Jacquey, C., Mazelle, C., d'Uston, C., Möbius, E., Kistler, L.M., Crocker, K., Granoff, M., Mouikis, C., Popecki, M., Vosbury, M., Klecker, B., Hovestadt, D., Kucharek, H., Kuenneth, E., Paschmann, G., Scholer, M., Sckopke, N., Seidenschwang, E., Carlson, C.W., Curtis, D.W., Ingraham, C., Lin, R.P., McFadden, J.P., Parks, G.K., Phan, 
T., Formisano, V., Amata, E., Bavassano-Cattaneo, M.B., Baldetti, P., Bruno, R., Chionchio, G., Di Lellis, A., Marcucci, M.F., Pallocchia, G., Korth, A., Daly, P.W., Graeve, B., Rosenbauer, H., Vasyliunas, V., McCarthy, M., Wilber, M., Eliasson, L., Lundin, R., Olsen, S., Shelley, E. G., Fuselier, S., Ghielmetti, A.G., Lennartsson, W., Escoubet, C.P., Balsiger, H., Friedel, R., Cao, J.-B., Kovrazhkin, R.A., Papamastorakis, I., Pellat, R., Scudder, J., and Sonnerup, B. (2001) First multispacecraft ion measurements in and near the Earth's magnetosphere with the identical Cluster ion spectrometry (CIS) experiment. Annales Geophysicae 19, 1303-1354.

Ribas, I., Guinan, E.F., Gudel, M., and Audard, M. (2005) Evolution of the solar activity over time and effects on planetary atmospheres I: Highenergy irradiances (1-1700 A). Astrophys. J. 622, 680-694.

Russell, C.T., Luhmann, J.G., and Strangeway, R.J. (2006) The solar wind interaction with Venus through the eyes of the Pioneer Venus Orbiter. Planet. Space Sci. 54, 1482-1495.

Sagan, C. and Chyba, C. (1997) The early faint Sun paradox: organic shielding of ultraviolet-labile greenhouse gases. Science 276, 1217-1221.

Sagan, C. and Mullen, G. (1972) Earth and Mars-evolution of atmospheres and surface temperatures. Science $177,52-56$.

Shaviv, N.J. (2003) Toward a solution to the early faint Sun paradox: a lower cosmic ray flux from a stronger solar wind. J. Geophys. Res. 108, 1437.

Sibeck, D.G., Paschmann, G., Treumann, R.A., Fuselier, S.A., Lennartsson, W., Lockwood, M., Lundin, R., Ogilvie, K.W., Onsager, T.G., Phan, T.-D., Roth, M., Scholer, M., Sckopke, N., Stasiewicz, K., and Yamauchi, M. (1999) Chapter 5-Plasma transfer processes at the magnetopause. Space Sci. Rev. 88, 207-283.

Stenbaek-Nielsen, H.C., Wescott, E.M., Rees, D., Valenzuela, A., and Brenning, N. (1990) Non-solar UV produced ions observed optically from the CRIT I critical velocity ionization experiment. J. Geophys. Res. 95, 7749-7757.

Sumita, I., Hatakeyama, T., Yoshihara, A., and Hamano, Y. (2001) Paleomagnetism of late Archean rocks of Hamersley basin, Western Australia and the paleointensity at early Proterozoic. Phys. Earth Planet. Inter. 128, 223-241.

Svensmark, H. and Friis-Christensen, E. (1997) Variation of cosmic ray flux and global cloud coverage. Journal of Atmospheric and Solar-Terrestrial Physics 59, 1225-1232.

Tian, F., Toon, O.B., Pavlov, A.A., and De Sterck, H. (2005) A hydrogen-rich early Earth atmosphere. Science 308, 1014-1017.

Tian, F., Toon, O.B., and Pavlov, A.A. (2006) Response to Comment on "A Hydrogen-Rich Early Earth Atmosphere." Science 311, 38.

Tinsley, B.A., Hodges, R.R., Jr., and Rohrbaugh, R.P. (1986) Monte Carlo models for the terrestrial exosphere over a solar cycle. J. Geophys. Res. 91, 13631-13647.

Vidal-Madjar, A. (1978) The earth hydrogen exobase near a solar minimum. Geophys. Res. Lett. 5, 29-32.
Wahlund, J.-E., Bostrom, R., Gustafsson, G., Gurnett, D.A., Kurth, W.S., Pedersen, A., Averkamp, T.F., Hospodarsky, G.B., Persoon, A.M., Canu, P., Neubauer, F.M., Dougherty, M.K., Eriksson, A.I., Morooka, M.W., Gill, R., Andre, M., Eliasson, L., and Muller-Wodarg, I. (2005) Cassini measurements of cold plasma in the ionosphere of Titan. Science 308, 986-989.

Wahlund, J.-E., Modolo, R., Eriksson, A.I., Kurth, W.S., Averkamp, T.F., Coates, A., Lewis, G.R., Bertucci, C., Doughterty, M.K., and Muller-Wodarg, I. (2006) Magnetosphere-ionosphere interaction and ionospheric escape from Titan: What can we learn from Cassini measurements. In Kiruna Mars Workshop Abstract Booklet, edited by S. Barabash, E. Carlsson, and H. Gunell, Kiruna, Sweden, pp. 56.

Wolff, R.S., Goldsrein, B.E., and Yeates, C.M. (1980) The onset and development of Kelvin-Helmholtz instability at the venusian ionopause. J. Geophys. Res. 85, 7697.

Wood, B.E. (2006) The solar wind and the Sun in the past. Space Sci. Rev. 126, 3-14.

Wood, B.E., Linsky, J.L., Muller, H.-R., and Zank, G.P. (2005) Inferences about the history of the solar wind from stellar wind measurement. In Proceedings of the Solar Wind 11 / SOHO 16, "Connecting Sun and Heliosphere" Conference, ESA SP-592, edited by B. Fleck, T.H. Zurbuchen and H. Lacoste, ESA Publications Division, Noordwijk, The Netherlands, pp. 387.

Yamauchi, M. and Lundin, R. (1997) The wave-assisted cusp model: comparison to low-altitude observations. Physics and Chemistry of the Earth 22, 729-734.

Yamauchi, M. and Lundin, R. (2001) Comparison of various cusp models with high- and low-resolution observations. Space Sci. Rev. 95, 457-468.

Yamauchi, M., Lundin, R., Norberg, O., Sandahl, I., Eliasson, L., and Winningham, D. (2003) Signature of direct magnetosheath plasma injections onto closed field-line regions based on observations at mid- and low-altitudes. In Earth's Low-Latitude Boundary Layer, edited by P. Newell and T. Onsager, AGU monograph, American Geophysical Union, Washington, DC, pp. 179-188.

Yamauchi, M., Eliasson, L., Lundin, R., and Norberg, O. (2005a) Unusual heavy ion injection events observed by Freja. Annales Geophysicae 23, 535-543.

Yamauchi, M., Sandahl, I., Nilsson, H., Lundin, R., and Eliasson, L. (2005b) Budget and roles of heavy ions in the Solar System. In Trends in Space Science and Cosmic Vision 2020, edited by F. Favata, J. Sanz-Forcada, A. Gimenez, European Space Agency Special Publication 588, 397-400.

Yamauchi, M., Futaana, Y., Fedorov, A., Dubinin, E., Lundin, R., Sauvaud, J.-A., Winningham, D., Frahm, R., Barabash, S., Holmstrom, M., Woch, J., Fraenz, M., Budnik, E., Borg, H., Sharber, A.J., Coates, Y., Soobiah, H., Koskinen, E., Kallio, K., Asamura, H., Hayakawa, C., Curtis, J.R., Hsieh, K.C., Sandel, B.R., Grande, M., Grigoriev, A., Wurz, P., Orsini, S., Brandt, P., McKenna-Lawler, S., Kozyra, J., and Luhmann, J. (2006) IMF direction derived from cycloid-like ion dis- 
tribution observed by Mars Express. Space Sci. Rev. 126, 239-266.

Zhang, M.H.G., Luhmann, J.G., Kliore, A.J., and Russell, C.T. (1990) A post-Pioneer Venus reassessment of the martian dayside ionosphere as observed by radio occultation methods. J. Geophys. Res. 95, 14829-14839.

Zhang, T.L., Luhmann, J.G., and Russell, C.T. (1991) The magnetic barrier at Venus. J. Geophys. Res. 96, 1114511153.
Address reprint requests to: M. Yamauchi Swedish Institute of Space Physics Box 812, SE-98128

Kiruna, Sweden

E-mail: M.Yamauchi@irf.se 